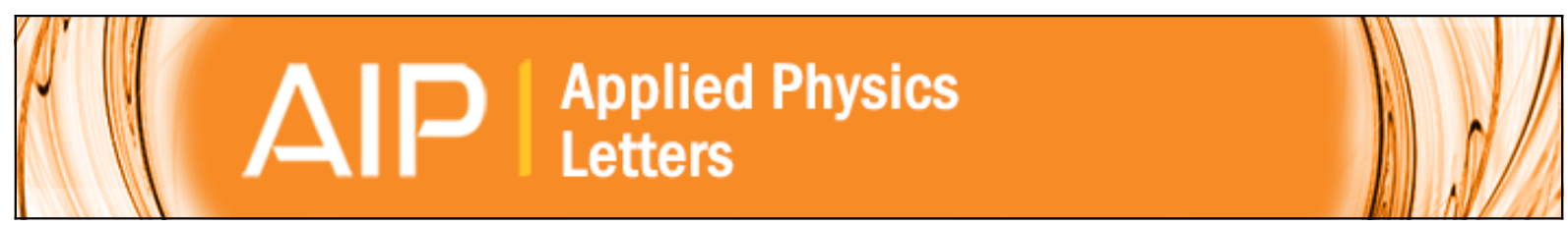

\title{
Cavity-enhanced optical detection of carbon nanotube Brownian motion
}

S. Stapfner, L. Ost, D. Hunger, J. Reichel, I. Favero, and E. M. Weig

Citation: Applied Physics Letters 102, 151910 (2013); doi: 10.1063/1.4802746

View online: http://dx.doi.org/10.1063/1.4802746

View Table of Contents: http://scitation.aip.org/content/aip/journal/apl/102/15?ver=pdfcov

Published by the AIP Publishing

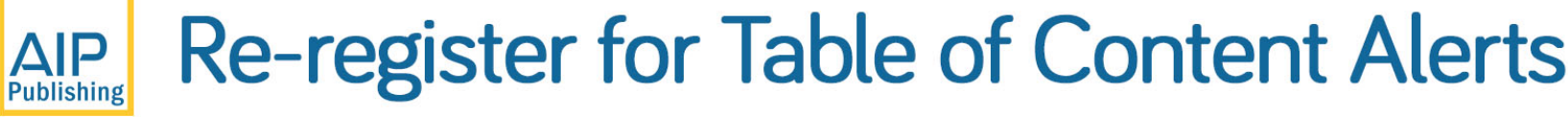

Create a profile.

Sign up today! 


\title{
Cavity-enhanced optical detection of carbon nanotube Brownian motion
}

\author{
S. Stapfner, ${ }^{1}$ L. Ost, ${ }^{1}$ D. Hunger, ${ }^{1}$ J. Reichel, ${ }^{2}$ I. Favero, ${ }^{3}$ and E. M. Weig ${ }^{1, a), b)}$ \\ ${ }^{1}$ Center for NanoScience and Fakultät für Physik, Ludwig-Maximilians-Universität München, \\ Geschwister-Scholl-Platz 1, 80539 München, Germany \\ ${ }^{2}$ Laboratoire Kastler Brossel, Ecole Normale Supérieure, Université Pierre et Marie Curie, \\ CNRS, 24 rue Lhomond, 75005 Paris, France \\ ${ }^{3}$ Laboratoire Matériaux et Phénomènes Quantiques, Université Paris-Diderot, Sorbonne Paris Cité, \\ CNRS, UMR 7162, 10 rue Alice Domon et Léonie Duquet, 75013 Paris, France
}

(Received 8 November 2012; accepted 8 April 2013; published online 17 April 2013)

\begin{abstract}
Optical cavities with small mode volume are well-suited to detect the vibration of sub-wavelength sized objects. Here we employ a fiber-based, high-finesse optical microcavity to detect the Brownian motion of a freely suspended carbon nanotube at room temperature under vacuum. The optical detection resolves deflections of the oscillating tube down to $70 \mathrm{pm} / \mathrm{Hz}^{1 / 2}$. A full vibrational spectrum of the carbon nanotube is obtained and confirmed by characterization of the same device in a scanning electron microscope. Our work extends the principles of high-sensitivity optomechanical detection to molecular scale nanomechanical systems. ( 2013 AIP Publishing LLC [http://dx.doi.org/10.1063/1.4802746]
\end{abstract}

Probing the vibrational motion of nano-scale objects has great potential for advancing next-generation technologies such as resonant mass or bio-sensing. ${ }^{1-3} \mathrm{~A}$ key example is carbon nanotubes (CNTs), which, due to their molecular scale mass and diameter, promise to display ultimate sensitivities and may even allow to resolve the quantum nature of mechanical vibration in optomechanical experiments. ${ }^{4,5}$ However, small vibrational amplitudes and dimensions complicate the realization of CNT-based mechanical devices. One fundamental challenge is to resolve the thermally excited Brownian motion of CNTs to enable operation of the undriven device in the linear regime and to limit the influence of spurious nonlinear effects observed in CNTs. ${ }^{6}$ Transmission electron microscopy (TEM) and scanning electron microscopy (SEM) have both been employed to visualize the thermal motion of CNTs, ${ }^{7,8}$ yielding a superposition of the small-amplitude envelopes of all oscillating modes. Electrical schemes are well-suited to detect vibrating CNTs but typically require a coherent actuation voltage ${ }^{2,9}$ and are typically not sensitive enough to resolve the Brownian motion. Dark field illumination was combined with confocal microscopy to detect the mechanical oscillation of driven CNTs with a diameter of $80 \mathrm{~nm}$; however, the Brownian motion amplitude could not be resolved with this approach. ${ }^{11}$ Ultra-sensitive optical detection technique ${ }^{10}$ are hindered by the diffraction limit, with typical tube diameters much smaller than the wavelength of visible light. However, even in the subwavelength regime, the sensitivity can be enhanced by using an optical cavity. ${ }^{12}$ In earlier work we employed a fiber-based optical micro-cavity of small mode volume and high finesse to measure the Brownian motion of an amorphous carbon based nanorod with a diameter of about $100 \mathrm{~nm} .{ }^{13}$ Following a proposal of cavity optomechanics with CNTs, ${ }^{12}$ in the present work the nanorod is replaced by a carbon nanotube with a ten times smaller

\footnotetext{
${ }^{a)}$ Present address: Department of Physics, Universität Konstanz, 78457 Konstanz, Germany.

b) Author to whom correspondence should be addressed. Electronic mail: eva.weig@uni-konstanz.de
}

diameter. Taking advantage of an improved cavity with significantly increased finesse, we present data clearly resolving the Brownian motion of the CNT oscillating within the cavity light field. Research in optomechanics ${ }^{14-17}$ will benefit from the in-cavity implementation of carbon nanotubes, which are the smallest solid-state mechanical resonators available to date, and have not yet been explored in this context.

The direct optical detection of a CNT of deep subwavelength dimensions is a challenging task. ${ }^{11}$ In an optical cavity, however, the dispersive and dissipative interactions between the nanotube and the light field are enhanced. Particularly for a high finesse cavity of small mode volume, the signature of the CNT displacement in the cavity response is amplified. For the case of a dispersive interaction, the CNT imprints a phase shift on the photons in the cavity, which results in a resonance frequency shift of the cavity mode. On the other hand, a dissipative interaction, for example, caused by absorption and scattering of photons, leads to decreasing the circulating optical power. The strength of both types of interaction depends on the position and orientation of the nanotube within the cavity mode. Thus by measuring the frequency and intensity of the cavity resonant response, information can be gained on these interactions. In the present work both dispersive and dissipative interaction of the CNT can contribute to the observed signal.

To detect the Brownian motion of the CNT, we employ a fiber-based microcavity formed by two opposing, concavely shaped fiber end facets coated with highly reflective Bragg mirrors $^{18,19}$ (Fig. 1(a)). For a mirror separation of $37 \mu \mathrm{m}$, the cavity optical mode waist radius is $3.4 \mu \mathrm{m}$, and the finesse is measured to be 24500 for a wavelength of $780 \mathrm{~nm}$. The CNT is doubly clamped and freely suspended across a gap in the substrate as indicated schematically in Fig. 1(a) and in the post-processed SEM micrograph of the investigated nanotube in Fig. 1(b). The suspended section of the CNT is $19.7 \mu \mathrm{m}$ long with a diameter between 6 and $8 \mathrm{~nm}$, consisting of 5 to 7 individual tubes forming a thin rope. The slightly wavy shape of the CNT indicates the absence of tensile stress.

For optical measurements of CNT vibration the substrate chip is introduced into the fiber-based cavity using a 
(a)

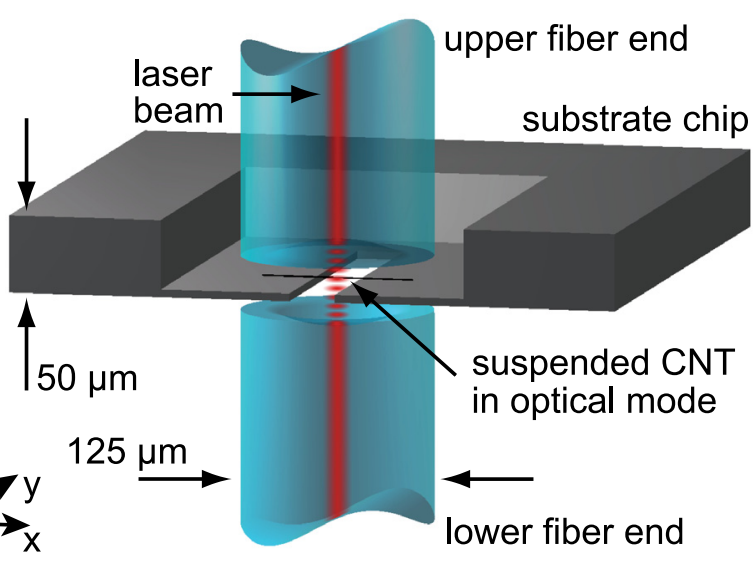

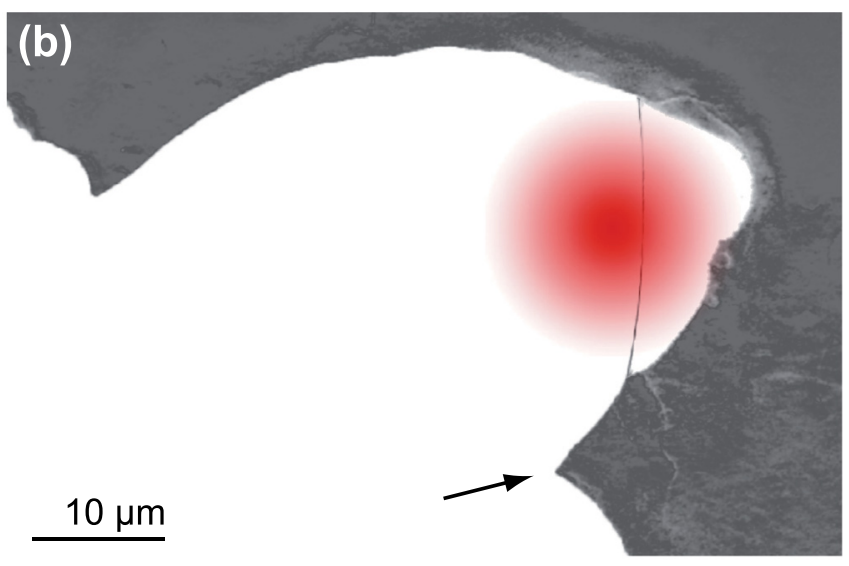

FIG. 1. Illustration of the experimental setup. (a) Schematic view of cavity and sample chip with the CNT introduced into the cavity mode. (b) Illustration based on an SEM image of the CNT under investigation suspended across the gap (white) with surrounding substrate (gray). The red spot illustrates the position and extension of the optical cavity mode during the experiment.

three axis XYZ positioner as illustrated in Fig. 1(a). In order to circumvent gas damping ${ }^{11}$ of the mechanical motion, the experiments are carried-out in a custom fiber-compliant vacuum cell at a pressure of $10^{-5}$ mbar and at room temperature. The cavity is pumped with a stabilized diode laser at $780 \mathrm{~nm}$. Details on fabrication and characterization of devices and on the experimental setup can be found in the supplementary material. ${ }^{20}$

The optical power transmitted on the empty cavity resonance is $0.56 \mu \mathrm{W}$. Bringing the CNT into the optical mode, as illustrated by the red spot in Fig. 1(b), the cavity transmission drops by $29 \%$. This drop is predominantly caused by residual clipping losses originating from the presence of the hosting substrate edges near the CNT. The CNT vibration is analyzed by probing the optical noise of the reflected light with a spectrum analyzer. When locked on the slope of the cavity resonance, the vibrational motion of the CNT is first characterized under external actuation from a drive piezo mounted below the substrate chip. Under white noise actuation ( $14 \mathrm{dBm}$ with $20 \mathrm{MHz}$ bandwidth), a series of resonances are clearly observed above the noise floor (Fig. 2(a) gray trace). Spectra shown in Figure 2(a) are obtained after substraction of the reference background of the empty cavity. The main peak at $0.51 \mathrm{MHz}$ is the fundamental flexural mode of the tube. This peak and other spectral features (black arrows) have been identified as mechanical resonances of the CNT through SEM experiments complemented by beam theory calculations and signal amplitude estimations, as detailed below. The main peak at $0.51 \mathrm{MHz}$ rises more than $20 \mathrm{~dB}$ above the noise floor, even when the piezo is not driven (Fig. 2(a) red data). A Lorentzian fit to the data points of the undriven resonance (Fig. 2(b) black line) yields a quality factor of 300. Interestingly, this signal peak is not stable in frequency but fluctuates slowly between $0.47 \mathrm{MHz}$ and $0.52 \mathrm{MHz}$ on the timescale of minutes. We suggest that slack and conformational instability of the tube, which are also observed in the SEM, contribute most to this behavior. Furthermore, a strong dependence of the signal amplitude on the position of the CNT in the optical mode is observed. The resonance vanishes completely when the CNT is positioned a few microns away from the optical mode axis. Further information is obtained when the sample is positioned such that the optical mode hits a protrusion of the hosting substrate (for example, at the position indicated by a black arrow in Fig. 1(b), about ten microns away from the CNT). With this protrusion entering the optical mode, the drop in cavity transmission is still of order $29 \%$, but the spectrum does not exhibit the resonance near $0.51 \mathrm{MHz}$. Instead a series of extra peaks below $0.22 \mathrm{MHz}$ appear most probably originating from vibrating substrate modes (see supplementary material ${ }^{20}$ ). Such substrate resonances are not observed above $0.22 \mathrm{MHz}$, corroborating that the main
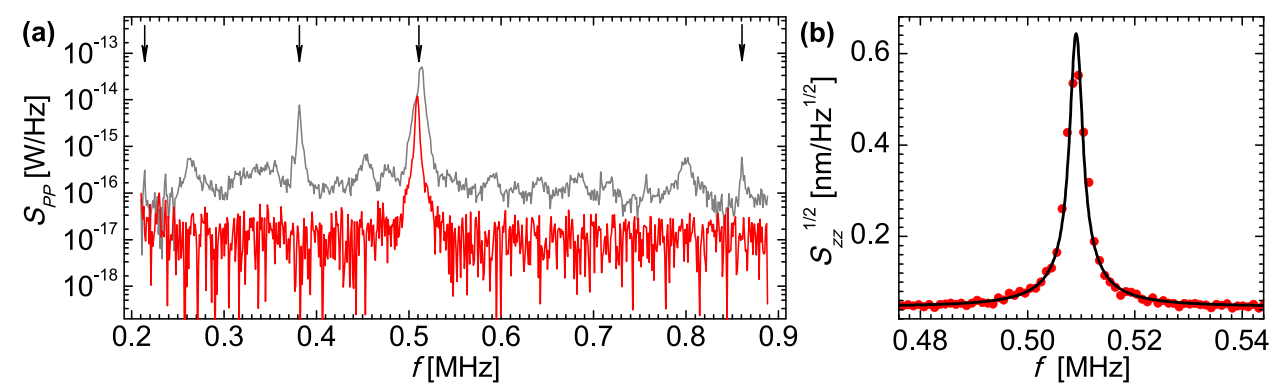

FIG. 2. (a) Power spectra with subtracted background reveal the CNT's vibrational spectrum. The gray trace is obtained with the CNT placed in the cavity mode, and $14 \mathrm{dBm}$ of white noise is applied to the drive piezo, whereas for the red trace the drive is switched off. The remaining peak near $0.51 \mathrm{MHz}$ rising more than $20 \mathrm{~dB}$ above the noise floor evidences the Brownian motion of the CNT. Black arrows point at peaks confirmed as mechanical resonances of the CNT by subsequent SEM experiments. (b) Zoom of the CNT's Brownian noise peak with Lorentzian fit (black) and data points calibrated to Brownian vibrational amplitudes. 
resonance at $0.51 \mathrm{MHz}$ does not stem from a substrate mode. The cavity resonant transmission exhibits constant values for the CNT being placed at several $z$-positions inside the cavity. The fact that the presence of the CNT does not lead to additional cavity losses is interpreted by a dominating influence of clipping losses to the dissipative signal component. This implies that the nanomechanical motion detection relies primarily on the dispersive interaction.

Conventional Euler Bernoulli theory allows for a satisfactory estimate of the resonance frequency $f_{0}=\omega_{0} /(2 \pi)$ of the fundamental flexural oscillation of the measured CNT. $^{8,21}$ The model of an unstressed beam ${ }^{22}$ yields

$$
\omega_{0}=\frac{\beta^{2}}{l^{2}} \sqrt{\frac{Y I}{\rho A}}
$$

where $\beta=4.72$ for the fundamental mode and $l$ is the length of the CNT. Values for Young's modulus $Y$ in the range between 0.3 and $1 \mathrm{TPa}$ can be found in literature. ${ }^{23-25}$ Based on TEM analysis we consider the observed CNT to be either a multiwall nanotube or a rope comprising five to seven individual tubes with a total outer diameter $d_{0}$ between 6 and $8 \mathrm{~nm}$. The area moment of inertia $I=\pi\left(d_{\mathrm{o}}^{4}-d_{\mathrm{i}}^{4}\right) / 64$ and the cross-section area $A=\pi\left(d_{\mathrm{o}}^{2}-d_{\mathrm{i}}^{2}\right) / 4$ are used for multiwall nanotubes with the inner diameter $d_{\mathrm{i}}=d_{\mathrm{o}}-n \Delta_{\mathrm{s}}$, the number of walls $n$, and the inter wall spacing $\Delta_{\mathrm{s}}=0.335 \mathrm{~nm}$ and for ropes with $d_{\mathrm{i}}=1 \mathrm{~nm}$. The physical mass density $\rho$ is inferred from the mass density of graphite $\rho_{\text {graphite }}=$ $2200 \mathrm{~kg} / \mathrm{m}^{3}$ along with $n, \Delta_{\mathrm{s}}$, and geometrical considerations for both cases. Entering the above input parameters into Eq. (1) yields an estimated resonance frequency between 0.16 and $0.63 \mathrm{MHz}$, which is consistent with the measured frequency of the CNT.

The equipartition theorem allows calibration of the cavity noise peak in Fig. 2(a) to the Brownian motional amplitude of the CNT using

$$
\frac{1}{2} k_{\mathrm{B}} T=\frac{1}{2} k\left\langle z^{2}\right\rangle,
$$

with Boltzmann constant $k_{\mathrm{B}}$, temperature $T=300 \mathrm{~K}$, and spring constant $k=\omega_{0}^{2} m_{\text {eff }}$. The mean squared amplitude $\left\langle z^{2}\right\rangle$ is the integral of the position squared spectral density in frequency space $\int_{0}^{\infty} S_{z z}(\omega) d \omega$, and the effective mass $m_{\mathrm{eff}}$ $=0.45 \mathrm{~m}=0.5 \pm 0.4 \mathrm{fg}$ for the fundamental mode $\operatorname{mos}^{20,26}$ is derived from the physical mass $m=\rho l A$ (see supplemental material $^{20}$ ). The large uncertainty in the mass stems from the above-mentioned uncertainty in the composition and diameter of the tube and translates into an error of $50 \%$ for the calibration shown in Fig. 2(b). The Brownian motion peak amplitude of the nanotube of $0.9 \mathrm{~nm} \pm 50 \%$ was measured with a sensitivity of $70 \mathrm{pm} / \mathrm{Hz}^{1 / 2} \pm 50 \%$.

Assuming a purely dispersive optomechanical interaction, the effect of the thermal displacement of the CNT on the cavity reflection is estimated using the dispersive cavity frequency shift ${ }^{27}$

$$
\frac{\delta \omega_{\mathrm{c}}}{\omega_{\mathrm{c}}}=-\frac{1}{2} \frac{\int \delta \mathbf{P}(\mathbf{r}) \cdot \mathbf{E}(\mathbf{r}) d^{3} \mathbf{r}}{\int \epsilon_{0} \mathbf{E}^{2}(\mathbf{r}) d^{3} \mathbf{r}}
$$

known from cavity perturbation theory, ${ }^{28}$ with electric field amplitude $\mathbf{E}(\mathbf{r})$, polarization $\delta \mathbf{P}(\mathbf{r})=\epsilon_{0}\left(\epsilon_{\mathrm{r}}-1\right) \mathbf{E}(\mathbf{r})$, dielectric constant $\epsilon_{0}$, and permittivity for CNTs ${ }^{29} \epsilon_{\mathrm{r}} \approx 3$. For the fundamental flexural mode of the investigated CNT and in the case of perfect alignment of the tube orthogonal to the $\mathrm{TEM}_{00}$ cavity mode axis, the integral of Eq. (3) leads to a maximal frequency-pull coupling rate $g_{\text {om }}=\partial \omega_{\mathrm{c}} / \partial z$ of $(2 \pi) 1 \mathrm{MHz} / \mathrm{nm}$ along the cavity axis. Assuming the dispersive detection is performed with the CNT centered between a node and antinode of the optical field, the employed laser power and detector response predict a peak cavity reflection noise power of $1 \mathrm{pW} / \mathrm{Hz}$ for the Brownian motion of the CNT. A more realistic estimate includes a finite tilt of the CNT with respect to the wavefronts of the cavity field. For an angle of $7^{\circ}$ consistent with the alignment tolerance under binocular inspection, a peak value of $10 \mathrm{fW} / \mathrm{Hz}$ is computed which agrees with the experimentally observed signal. This independently supports the identification of the $0.51 \mathrm{MHz}$ resonance as Brownian motion of the fundamental flexural mode.

In addition, SEM analysis on the suspended CNT sample is performed to substantiate this interpretation. This investigation starts after completion of the optical cavity measurements, in order to avoid electron beam induced contamination. As shown in Fig. 3(a), the CNT is still present at its original position and appears visually unchanged after being exposed to the intense light field of the cavity $\left(\approx 24 \mathrm{~kW} \mathrm{~cm}^{-2}\right)$. In order to visualize the CNT vibrational modes in the SEM, external actuation through the drive piezo is employed. Using the same white noise signal as applied in Fig. 2(a) the CNT image is found to blur, showing the superposition of the envelopes of the simultaneously excited flexural oscillation modes ${ }^{8}$ (Fig. 3(b)). Driving the sample with a sinusoidal signal allows to isolate individual vibrational modes in the range between 0.1 and $1 \mathrm{MHz}$. This allows identifying all peaks from the optical cavity spectrum marked by black arrows in Fig. 2(a) and supplementary Fig. S9 as CNT mechanical modes ${ }^{20}$ at $0.153 \mathrm{MHz}, 0.168 \mathrm{MHz}$, $0.215 \mathrm{MHz}, 0.377 \mathrm{MHz}, 0.51 \mathrm{MHz}, 0.864 \mathrm{MHz}, 0.907 \mathrm{MHz}$, and $0.923 \mathrm{MHz}$. Figure 3(c) shows the oscillation of the fundamental flexural mode of the CNT when driven by a $14 \mathrm{dBm}$ power sinusoidal signal at $0.51 \mathrm{MHz}$. Measuring the

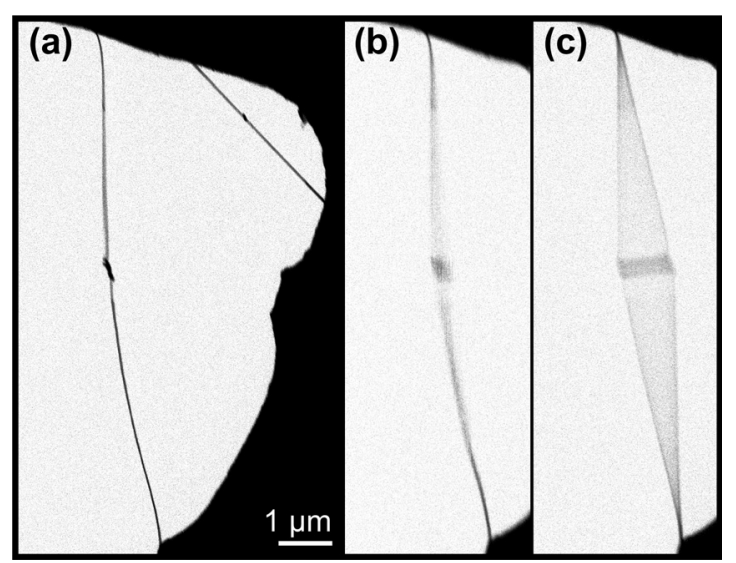

FIG. 3. SEM images showing the CNT (a) at rest and excited through the drive piezo with $14 \mathrm{dBm}$ (b) of white noise and (c) at $0.51 \mathrm{MHz}$. Colors are inverted such that the substrate and CNT appear black and the gap is light gray. 
vibrational amplitude and sweeping the drive frequency across this resonance at much lower drive powers allows to estimate the quality factor to be about $250 \pm 50$ which comes close to the value measured in the cavity. It has to be noted that electron beam induced deposition on the CNT affects the mechanical properties, but by exposing the tube to the electron beam only at small areas during frequency scanning and taking single shot images this effect is minimized such that no obvious down-shift in resonance frequency has been observed.

One possible effect distorting the calibration of the CNT Brownian amplitude is heating of the CNT by absorption of laser light. Neglecting optical losses by clipping on the hosting substrate and scattering losses induced by the $\mathrm{CNT}$, we envision the extreme case where all optical power lost in the cavity is absorbed by the CNT and turns into heat. Heat generated in the illuminated section of the CNT (cf., Fig. 1(b)) propagates along the CNT to the clamps and dissipates into the thermal bath of the substrate. Using a heat transfer model for the heat flow $\dot{Q}=\kappa A \Delta T / l$ with the thermal conductivity ${ }^{30} \kappa=118 \mathrm{~W} /(\mathrm{mK})$ of a comparable CNT of length $l$ and cross-section $A$ yields an increase in temperature $\Delta T$ of $150 \mathrm{~K}$. Under the above extreme assumptions the CNT would oscillate at an elevated vibrational temperature with larger Brownian amplitude, entailing a correction of the measurement calibration by a factor 1.2 , which is smaller than the uncertainty coming from the CNT mass estimation.

Furthermore optomechanical back-action could actuate the CNT and modify the mechanical resonance and linewidth. ${ }^{12}$ However, an estimate of the maximum increase in vibrational temperature due to dynamical backaction ${ }^{31}$ yields at most a few kelvins and is thus negligible in our experiment. Further, we extract similar Q-values from measurements in the cavity and in the SEM, strongly indicating that the CNT is not driven optomechanically.

Being the smallest solid-state mechanical resonators available to date along with their relatively large zero point amplitude $z_{\text {zpf }}=\left(\hbar /\left(2 m_{\text {eff }} \omega_{0}\right)\right)^{1 / 2}$ makes CNTs an interesting candidate for optomechanical studies. For the tube investigated in the present article $z_{\mathrm{zpf}}=8.2 \mathrm{pm}$. With an improved yet technically feasible cavity line width $\kappa_{\mathrm{c}} /(2 \pi)=20 \mathrm{MHz}$ and for good alignment of the CNT to the optical mode, a zero point equivalent optomechanical coupling rate $g_{0}=g_{\text {om }} z_{\text {zpf }}=(2 \pi) 8.2 \mathrm{kHz}$ is expected. A cryogenic environment can boost the mechanical quality factor of the $\mathrm{CNT}^{9}$ beyond $10^{5}$, which would place the system deeply into the strong coupling regime. ${ }^{32,33}$ This is apparent form the cooperativity $C=4 g_{0}^{2} n_{\mathrm{c}} Q /\left(\kappa_{\mathrm{c}} \omega_{0}\right)$, an important figure of merit for optomechanical systems, which relates the zero point optomechanical coupling rate $g_{0}$, the intracavity photon number $n_{\mathrm{c}}$, the cavity linewidth $\kappa_{\mathrm{c}}$, and the mechanical damping rate $Q / \omega_{0}$. For the described parameters a cooperativity per photon $C_{0}=C / n_{\mathrm{c}}=2.7$ can be expected, which is higher than that reported in state-of-the-art optomechanical experiments. $^{32,33}$

In summary, we have investigated freely suspended carbon nanotubes inserted into a fiber-based optical micro cavity. Taking advantage of the small mode volume of the cavity we clearly resolve the Brownian motion of a CNT, a mechanical nanostructure much smaller than the wavelength of the employed light. Optically measured mechanical resonance frequencies are confirmed by measurements in the scanning electron microscope. In contrast to SEM imaging techniques, the optical detection technique presented here allows much higher motional sensitivity, integration in fiber optics devices, and does not contaminate the CNT. Furthermore we anticipate these advances to enable optomechanical carbon nanotube architectures that will allow probing of the quantum nature of molecular mechanical systems.

We thank C. Schönenberger and M. Weiss for help in setting up clean CNT growth, M. Döblinger for TEM analysis and imaging, J. P. Kotthaus and K. Karrai for fruitful discussions, and D. R. Southworth for critically reading the manuscript. We acknowledge financial support from the German-Israeli Foundation (GIF), the German Excellence Initiative via the Nanosystems Initiative Munich (NIM), the German/French Academic Exchange Service (DAAD/ EGIDE Procope program), and the Bayerisch-Französisches Hochschulzentrum (BFHZ).

${ }^{1}$ K. Jensen, K. Kim, and A. Zettl, Nat. Nanotechnol. 3, 533 (2008).

${ }^{2}$ J. Chaste, A. Eichler, J. Moser, G. Ceballos, R. Rurali, and A. Bachtold, Nat. Nanotechnol. 7, 301 (2012).

${ }^{3}$ M. S. Hanay, S. Kelber, A. K. Naik, D. Chi, S. Hentz, E. C. Bullard, E. Colinet, L. Duraffourg, and M. L. Roukes, Nat. Nanotechnol. 7, 602 (2012).

${ }^{4}$ I. Wilson-Rae, C. Galland, W. Zwerger, and A. Imamoglu, New J. Phys. 14, 115003 (2012).

${ }^{5}$ B. H. Schneider, S. Etaki, H. S. J. van der Zant, and G. A. Steele, e-print arXiv:1209.1514[cond-mat.mes-hall].

${ }^{6}$ A. Eichler, J. Moser, J. Chaste, M. Zdrojek, I. Wilson-Rae, and A. Bachtold, Nat. Nanotechnol. 6, 339 (2011).

${ }^{7}$ M. M. J. Treacy, T. W. Ebbesen, and J. M. Gibson, Nature (London) 381, 678 (1996).

${ }^{8}$ B. Babić, J. Furer, S. Sahoo, S. Farhangfar, and C. Schönenberger, Nano Lett. 3, 1577 (2003).

${ }^{9}$ G. A. Steele, A. K. Hüttel, B. Witkamp, M. Poot, H. B. Meerwaldt, L. P. Kouwenhoven, and H. S. J. van der Zant, Science 325, 1103 (2009).

${ }^{10}$ O. Arcizet, P.-F. Cohadon, T. Briant, M. Pinard, A. Heidmann, J.-M. Mackowski, C. Michel, L. Pinard, O. Français, and L. Rousseau, Phys. Rev. Lett. 97, 133601 (2006).

${ }^{11}$ S. Fukami, T. Arie, and S. Akita, Jpn. J. Appl. Phys. 48, 06FG04 (2009).

${ }^{12}$ I. Favero and K. Karrai, New J. Phys. 10, 095006 (2008).

${ }^{13}$ I. Favero, S. Stapfner, D. Hunger, P. Paulitschke, J. Reichel, H. Lorenz, E. M. Weig, and K. Karrai, Opt. Express 17, 12813 (2009).

${ }^{14}$ T. J. Kippenberg and K. J. Vahala, Science 321, 1172 (2008).

${ }^{15}$ F. Marquardt and S. M. Girvin, Physics 2, 40 (2009).

${ }^{16}$ I. Favero and K. Karrai, Nat. Photonics 3, 201 (2009).

${ }^{17}$ M. Aspelmeyer, S. Gröblacher, K. Hammerer, and N. Kiesel, J. Opt. Soc. Am. B 27, A189 (2010).

${ }^{18}$ D. Hunger, T. Steinmetz, Y. Colombe, C. Deutsch, T. W. Hänsch, and J. Reichel, New J. Phys. 12, 065038 (2010).

${ }^{19}$ N. E. Flowers-Jacobs, S. W. Hoch, J. C. Sankey, A. Kashkanova, A. M. Jayich, C. Deutsch, J. Reichel, and J. G. E. Harris, Appl. Phys. Lett. 101, 221109 (2012).

${ }^{20}$ See supplementary material at http://dx.doi.org/10.1063/1.4802746 for details on substrate fabrication and characterization, experimental setup, and methods along with further data and calculus for the effective mass.

${ }^{21}$ M. J. Martin and B. H. Houston, Appl. Phys. Lett. 91, 103116 (2007).

${ }^{22}$ W. Weaver, S. P. Timoshenko, and D. H. Young, Vibration Problems in Engineering, 5th ed. (John Wiley \& Sons, 1990).

${ }^{23}$ J.-P. Salvetat, G. A. D. Briggs, J.-M. Bonard, R. R. Bacsa, A. J. Kulik, T. Stöckli, N. A. Burnham, and L. Forró, Phys. Rev. Lett. 82, 944 (1999).

${ }^{24}$ R. S. Ruoff, D. Qian, and W. K. Liu, C. R. Phys. 4, 993 (2003). 
${ }^{25}$ M. Löffler, U. Weissker, T. Mühl, T. Gemming, and B. Büchner, Ultramicroscopy 111, 155 (2011).

${ }^{26}$ K. L. Ekinci and M. L. Roukes, Rev. Sci. Instrum. 76, 061101 (2005).

${ }^{27}$ D. E. Chang, C. A. Regal, S. B. Papp, D. J. Wilson, J. Ye, O. Painter, H. J. Kimble, and P. Zoller, Proc. Natl. Acad. Sci. U.S.A. 107, 1005 (2010) and supplementary information therein.

${ }^{28}$ R. A. Waldron, Proc. IEE Part C 107, 272 (1960).

${ }^{29}$ W. A. de Heer, W. S. Bacsa, A. Chatelain, T. Gerfin, R. Humphrey-Baker, L. Forro, and D. Ugarte, Science 268, 845 (1995).
${ }^{30}$ I.-K. Hsu, M. T. Pettes, A. Bushmaker, M. Aykol, L. Shi, and S. B. Cronin, Nano Lett. 9, 590 (2009).

${ }^{31}$ C. Metzger, I. Favero, A. Ortlieb, and K. Karrai, Phys. Rev. B 78, 035309 (2008).

${ }^{32}$ J. D. Teufel, D. Li, M. S. Allman, K. Cicak, A. J. Sirois, J. D. Whittaker, and R. W. Simmonds, Nature (London) 471, 204 (2011).

${ }^{33}$ A. H. Safavi-Naeini, T. P. M. Alegre, J. Chan, M. Eichenfield, M. Winger, Q. Lin, J. T. Hill, D. E. Chang, and O. Painter, Nature (London) 472, 69 (2011). 